\title{
CONVEXITY AND GENERALIZED BERNSTEIN POLYNOMIALS
}

\author{
by TIM N. T. GOODMAN, HALIL ORUÇ* and GEORGE M. PHILLIPS
}

(Received 28th May 1997)

Dedicated to S. L. Lee

In a recent generalization of the Bernstein polynomials, the approximated function $f$ is evaluated at points spaced at intervals which are in geometric progression on $[0,1]$, instead of at equally spaced points. For each positive integer $n$, this replaces the single polynomial $B_{n} f$ by a one-parameter family of polynomials $B_{n}^{q} f$, where $0<q \leq 1$. This paper summarizes briefly the previously known results concerning these generalized Bernstein polynomials and gives new results concerning $B_{n}^{q} f$ when $f$ is a monomial. The main results of the paper are obtained by using the concept of total positivity. It is shown that if $f$ is increasing then $B_{n}^{4} f$ is increasing, and if $f$ is convex then $B_{n}^{q} f$ is convex, generalizing well known results when $q=1$. It is also shown that if $f$ is convex then, for any positive integer $n, B_{n} f \leq B_{n}^{q} f$ for $0<q \leq r \leq 1$. This supplements the well known classical result that $f \leq B_{n} f$ when $f$ is convex.

1991 Mathematics subject classification: 41 A10.

\section{Introduction}

In this paper we discuss further properties of the generalized Bernstein polynomials defined by

$$
B_{n}(f ; x)=\sum_{r=0}^{n} f_{r}\left[\begin{array}{l}
n \\
r
\end{array}\right] x^{r} \prod_{s=0}^{n-r-1}\left(1-q^{s} x\right)
$$

where an empty product denotes 1 and $f_{r}=f([r] /[n])$. It is necessary to explain the notation. The function $f$ is evaluated at the ratios of the $q$-integers $[r]$ and $[n]$, where $q$ is a positive real number and

$$
[r]=\left\{\begin{array}{lr}
\left(1-q^{r}\right) /(1-q), & q \neq 1 \\
r, & q=1 .
\end{array}\right.
$$

We then define the $q$-factorial $[r]$ ! by

$$
[r] !=\left\{\begin{array}{lrl}
{[r] .[r-1] \ldots[1],} & r=1,2, \ldots, \\
1, & r & =0
\end{array}\right.
$$

- Supported by Dokuz Eylūl University, Izmir, Turkey. 
and the $q$-binomial coefficient $\left[\begin{array}{l}n \\ r\end{array}\right]$ by

$$
\left[\begin{array}{l}
n \\
r
\end{array}\right]=\frac{[n] !}{[r] ![n-r] !}
$$

for integers $n \geq r \geq 0$. These $q$-binomial coefficients satisfy the recurrence relations

$$
\left[\begin{array}{l}
n \\
r
\end{array}\right]=q^{n-r}\left[\begin{array}{l}
n-1 \\
r-1
\end{array}\right]+\left[\begin{array}{c}
n-1 \\
r
\end{array}\right]
$$

and

$$
\left[\begin{array}{l}
n \\
r
\end{array}\right]=\left[\begin{array}{l}
n-1 \\
r-1
\end{array}\right]+q^{r}\left[\begin{array}{c}
n-1 \\
r
\end{array}\right]
$$

We note from the above recurrence relations that $\left[\begin{array}{l}n \\ r\end{array}\right]$ is positive for $n \geq r \geq 0$ and all $q \geq 0$. It is then clear from (1.1) that if $f$ is positive on $[0,1]$ then, for all $q$ such that $0<q \leq 1$, $B_{n} f$ is positive on $[0,1]$. It is also easily verified that $B_{n}(f ; 0)=f(0), B_{n}(f ; 1)=f(1)$ and $B_{n}(f ; x)=f(x), 0 \leq x \leq 1$, when $f(x)$ is a polynomial of degree 1 or less.

In [4] there is a discussion of convergence and a Voronovskaya theorem on the rate of convergence, and a de Casteljau algorithm is given in [5] for computing $B_{n}(f ; x)$ recursively. In [3] it is shown that, if $f$ is convex,

$$
B_{n}(f ; x) \leq B_{n-1}(f ; x), \quad 0 \leq x \leq 1,
$$

for $n>1$ and $0<q \leq 1$.

This paper is concerned with the behaviour of the generalized Bernstein polynomials as $q$ varies. When we need to emphasize the dependence on $q$ we will write $B_{n}^{q}(f ; x)$ in place of $B_{n}(f ; x)$. In Section 2 we discuss the Bernstein polynomials for the monomials, which have a particularly simple form. In Section 3 we quote some results on the theory of total positivity which are used in the following sections. In Section 4 we discuss a change of basis, in order to show later how $B_{n}(f ; x)$ varies with the parameter q. Finally it is proved for all $n \geq 1$ and $0<q \leq 1$ that if $f$ is increasing, $B_{n}^{q} f$ is increasing, and if $f$ is convex then $B_{n}^{q} f$ is convex. We also show that if $f$ is convex on $[0,1]$ then $B_{n}^{\prime} f \leq B_{n}^{q} f$ for $0<q \leq r \leq 1$.

\section{The monomials}

We require some preliminaries. For any real function $f$ we define $\Delta^{0} f_{i}=f_{i}$ for $i=0,1, \ldots n$ and, recursively, 


$$
\Delta^{k+1} f_{i}=\Delta^{k} f_{i+1}-q^{k} \Delta^{k} f_{i}
$$

for $k=0,1, \ldots, n-i-1$, where $f_{i}$ denotes $f([i] /[n])$. It is easily shown by induction on $k$ that $q$-differences satisfy the relation

$$
\Delta^{k} f_{i}=\sum_{r=0}^{k}(-1)^{r} q^{r(r-1) / 2}\left[\begin{array}{l}
k \\
r
\end{array}\right] f_{i+k \rightarrow r}
$$

see Schoenberg [6], Lee and Phillips [2]. The generalized Bernstein polynomial (1.1) may also be written in the $q$-difference form (see [4])

$$
B_{n}(f ; x)=\sum_{j=0}^{n}\left[\begin{array}{l}
n \\
j
\end{array}\right] \Delta^{j} f_{0} x^{j}
$$

We now express the $q$-binomial coefficients as

$$
\left[\begin{array}{l}
n \\
j
\end{array}\right]=\frac{[n]^{j}}{[j] ! q^{j(j-1) / 2}} \pi_{j}^{n}, \quad 0 \leq j \leq n,
$$

where

$$
\pi_{j}^{n}=\prod_{r=0}^{j-1}\left(1-\frac{[r]}{[n]}\right)
$$

and an empty product denotes 1. It follows from (2.2) that $B_{n}\left(x^{i} ; x\right)$ is a polynomial of degree less or equal to $\min (i, n)$ and, using (2.2), (2.1) and (2.3), we obtain

$$
B_{n}\left(x^{i} ; x\right)=\sum_{j=0}^{i} \pi_{j}^{n}[n]^{j-i} S_{q}(i, j) x^{j}
$$

where

$$
S_{q}(i, j)=\frac{1}{[j] ! q^{j(j-1) / 2}} \sum_{r=0}^{j}(-1)^{r} q^{r(r-1) / 2}\left[\begin{array}{l}
j \\
r
\end{array}\right][j-r]^{i}
$$

We may verify by induction on $i$ that

$$
S_{q}(i+1, j)=S_{q}(i, j-1)+[j] S_{q}(i, j)
$$

for $i \geq 0$ and $j \geq 1$ with $S_{q}(0,0)=1, S_{q}(i, 0)=0$ for $i>0$ and we define $S_{q}(i, j)=0$ for $j>i$. We call $S_{q}(i, j)$ the Stirling polynomials of the second kind since when $q=1$ they are the Stirling numbers of the second kind. The recurrence relation (2.6) shows that, 
for $q>0$, the Stirling polynomials are polynomials in $q$ with non-negative integer coefficients and so are positive monotonic increasing functions of $q$. Thus $B_{n}\left(x^{i} ; x\right)$ and all its derivatives are non-negative on $[0,1]$. In particular, $B_{n}\left(x^{i} ; x\right)$ is convex. In Section 4 , we will find that, more generally, $B_{n}(f ; x)$ is convex when $f$ is convex.

\section{Total positivity}

In this section we will cite some results concerning totally positive matrices, which we require later to verify the shape-preserving properties of the generalized Bernstein polynomials.

Definition 3.1. For any real sequence $v$, finite or infinite, we denote by $S^{-}(v)$ the number of strict sign changes in $v$.

We use the same notation to denote sign changes in a function, as follows.

Definition 3.2. For a real-valued function $f$ on an interval $I$, we define $S^{-}(f)$ to be the number of sign changes of $f$, that is

$$
S^{-}(f)=\sup S^{-}\left(f\left(x_{0}\right), \ldots, f\left(x_{m}\right)\right)
$$

where the supremum is taken over all increasing sequences $\left(x_{0}, \ldots, x_{m}\right)$ in $I$ for all $m$.

Definition 3.3. We say that a matrix $\mathbf{A}=\left(a_{i j}\right)$ is $m$-banded if, for some $l, a_{i j} \neq 0$ implies $l \leq j-i \leq l+m$.

Definition 3.4. A matrix is said to be totally positive if all its minors are nonnegative.

It is easily verified that, with $0<x_{0}<x_{1}<\ldots<x_{n}$ the $(n+1) \times(n+1)$ Vandermonde matrix whose $(i, j)$ th element is $x_{i}^{j}, 0 \leq i, j \leq n$, is totally positive.

Theorem 3.1. A finite matrix is totally positive if and only if it is a product of 1banded matrices with non-negative elements.

Theorem 3.2 (Variation diminishing property). If $\mathbf{T}$ is a totally positive matrix and $\mathrm{v}$ is any vector for which $\mathrm{Tv}$ is defined, then $S^{-}(\mathrm{Tv}) \leq S^{-}(\mathrm{v})$.

Definition 3.5. We say that a sequence $\left(\phi_{0}, \ldots, \phi_{n}\right)$ of real-valued functions on an interval $I$ is totally positive if, for any points $x_{0}<\ldots<x_{n}$ in $I$, the collocation matrix $\left(\phi_{j}\left(x_{i}\right)\right)_{i, j=0}^{n}$ is totally positive.

Theorem 3.3. If $\left(\phi_{0}, \ldots, \phi_{n}\right)$ is totally positive on I then, for any numbers $a_{0}, \ldots, a_{n}$, 


$$
S^{-}\left(a_{0} \phi_{0}+\ldots+a_{n} \phi_{n}\right) \leq S^{-}\left(a_{0}, \ldots, a_{n}\right) .
$$

For the proofs of these theorems see [1].

Thus, from the total positivity of the Vandermonde matrix, we see that $\left(1, x, \ldots, x^{n}\right)$ is totally positive in any subinterval of $[0, \infty)$. On making the change of variable $t=x /(1-x)$, noting that $t$ is increasing function of $x$, we see that

$$
\left(1, x /(1-x), x^{2} /(1-x)^{2}, \ldots, x^{n} /(1-x)^{n}\right)
$$

is totally positive on $[0,1]$ and thus

$$
\left((1-x)^{n}, x(1-x)^{n-1}, x^{2}(1-x)^{n-2}, \ldots, x^{n}\right)
$$

is totally positive on $[0,1]$. For some $0<q \leq 1, n \geq 1, j=0, \ldots, n$, let

$$
P_{j}^{n, q}(x)=x^{j} \prod_{s=0}^{n-j-1}\left(1-q^{s} x\right), \quad 0 \leq x \leq 1,
$$

denote the functions which appear in the generalized Bernstein polynomials (1.1). We have seen above that

$$
\left(P_{0}^{n, 1}, P_{1}^{n, 1}, \ldots, P_{n}^{n, 1}\right)
$$

is totally positive on $[0,1]$ and we will see in Section 4 that the same is true of $\left(P_{0}^{n, q}, P_{1}^{n, q}, \ldots, P_{n}^{n, q}\right)$ for any $q, 0<q \leq 1$.

\section{Change of basis}

In this section we present results which will be used to show how $B_{n}(f ; x)$ varies with the value of the parameter $q$.

Since the functions defined in (3.1) are a basis for the subspace of the polynomials of degree at most $n$ then, for any $q, r, 0<q, r \leq 1$, there exists a non-singular matrix $T^{n, q, r}$ such that

$$
\left[\begin{array}{c}
P_{0}^{n, q}(x) \\
\vdots \\
P_{n}^{n, q}(x)
\end{array}\right]=\mathrm{T}^{n, q, r}\left[\begin{array}{c}
P_{0}^{n, r}(x) \\
\vdots \\
P_{n}^{n, r}(x)
\end{array}\right]
$$

Theorem 4.1. For $0<q \leq r$ all elements of the matrix $\mathrm{T}^{n, q, r}$ are non-negative.

Proof. We use induction on $n$. The result holds for $n=1$ since $T^{1, q, r}$ is the $2 \times 2$ identity matrix. Let us assume the result holds for some $n \geq 1$. Then, since 
184 TIM N. T. GOODMAN, HALIL ORUÇ AND GEORGE M. PHILLIPS

$$
P_{j+1}^{n+1, q}(x)=x P_{j}^{n, q}(x), \quad 0 \leq j \leq n,
$$

we have

$$
\left[\begin{array}{c}
P_{1}^{n+1, q}(x) \\
\vdots \\
P_{n+1}^{n+1, q}(x)
\end{array}\right]=\mathrm{T}^{n, q, r}\left[\begin{array}{c}
P_{1}^{n+1, r}(x) \\
\vdots \\
P_{n+1}^{n+1, r}(x)
\end{array}\right]
$$

Also, we have

$$
\begin{aligned}
P_{0}^{n+1, q}(x) & =(1-x) \ldots\left(1-q^{n-1} x\right)\left(1-q^{n} x\right) \\
& =\left(1-q^{n} x\right) \sum_{j=0}^{n} T_{0, j}^{n, q, r} P_{j}^{n, r}(x) .
\end{aligned}
$$

On substituting

$$
\left(1-q^{n} x\right) P_{j}^{n, r}(x)=P_{j}^{n+1, r}(x)+\left(r^{n-j}-q^{n}\right) P_{j+1}^{n+1, r}(x)
$$

and simplifying, we obtain

$$
\begin{aligned}
P_{0}^{n+1, q}(x)= & T_{0,0}^{n, q, r} P_{0}^{n+1, r}(x)+\left(1-q^{n}\right) T_{0, n}^{n, q, r} P_{n+1}^{n+1, r}(x) \\
& +\sum_{j=1}^{n}\left(\left(r^{n+1-j}-q^{n}\right) T_{0, j-1}^{n, q, r}+T_{0, j}^{n, q, r}\right) P_{j}^{n+1, r}(x) .
\end{aligned}
$$

Combining (4.1) and (4.2), we have

$$
\left[\begin{array}{c}
P_{0}^{n+1, q}(x) \\
P_{1}^{n+1, q}(x) \\
\vdots \\
P_{n+1}^{n+1, q}(x)
\end{array}\right]=\left[\begin{array}{cc}
T_{0,0}^{n, q, r} & \mathbf{v}_{n+1}^{T} \\
& \\
& \\
\mathbf{0} & \mathbf{T}^{n, q, r}
\end{array}\right]\left[\begin{array}{c}
P_{0}^{n+1, r}(x) \\
P_{1}^{n+1, r}(x) \\
\vdots \\
P_{n+1}^{n+1, r}(x)
\end{array}\right],
$$

where the elements of the row vector $\mathbf{v}_{n+1}^{T}$ are the coefficients of $P_{1}^{n+1, r}(x), \ldots, P_{n+1}^{n+1, r}(x)$ given by (4.2). Thus $T^{n+1, q, r}$ is the matrix in block form in (4.3) which, together with (4.2), shows that all elements of $T^{n+1, q, r}$ are non-negative. This completes the proof.

We now show that $\mathbf{T}^{\text {n,q,r }}$ can be factorized as a product of 1-banded matrices. First we require the following lemma.

Lemma 4.1. For $m \geq 1$ and $r, a \in \mathbf{R}$, let $\mathbf{A}(m, a)$ denote the $m \times(m+1)$ matrix 


$$
\left[\begin{array}{ccccc}
1 & r^{m}-a & & & \\
& 1 & r^{m-1}-a & & \\
& & \ddots & \ddots & \\
& & & 1 & r-a
\end{array}\right]
$$

Then

$$
\mathbf{A}(m, a) \mathbf{A}(m+1, b)=\mathbf{A}(m, b) \mathbf{A}(m+1, a)
$$

Proof. For $i=0, \ldots, m-1$ the $i$ th row of each side of (4.4) is

$$
\left[0, \ldots, 0,1, r^{m+1-i}+r^{m-i}-a-b,\left(r^{m-i}-a\right)\left(r^{m-i}-b\right), 0, \ldots, 0\right] .
$$

Theorem 4.2. For $n \geq 2$ and any $q, r$ the matrix $\mathbf{T}^{n, q, r}$ is given by the product

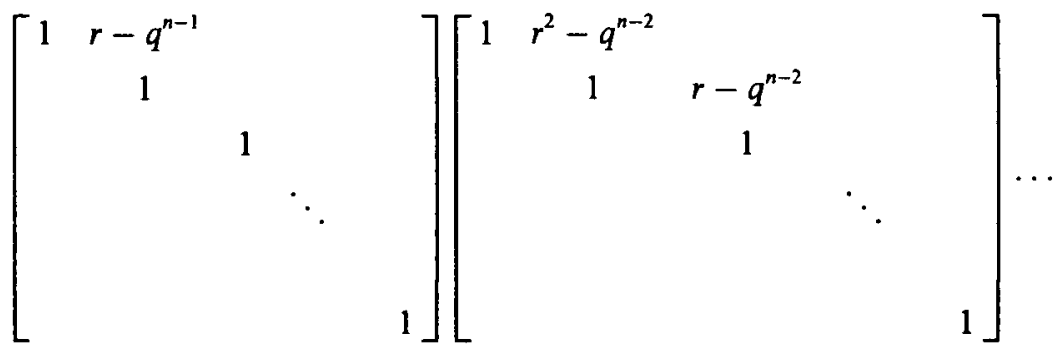

$$
\begin{aligned}
& {\left[\begin{array}{ccccccc}
1 & r^{n-1}-q & & & & & \\
& 1 & r^{n-2}-q & & & & \\
& & & & & & \\
& & & \ddots & \ddots & & \\
& & & & 1 & r-q & \\
& & & & 1 & \\
& & & & & 1
\end{array}\right]}
\end{aligned}
$$

Proof. We use induction on $n$. The result holds for $n=2$. Denote the above product by $\mathbf{S}^{n, q, r}$ and assume that, for some $n \geq 2, \mathbf{T}^{n, q, r}=\mathbf{S}^{n, q, r}$. Then we can express $\mathbf{S}^{n+1, q, r}$ as the product, in block form,

$$
\mathbf{S}^{n+1, q, r}=\left[\begin{array}{cc}
1 & \mathbf{c}_{0}^{T} \\
\mathbf{0} & \mathbf{I}
\end{array}\right]\left[\begin{array}{ll}
1 & \mathbf{c}_{1}^{T} \\
\mathbf{0} & \mathbf{B}_{1}
\end{array}\right]\left[\begin{array}{ll}
1 & \mathbf{c}_{2}^{T} \\
\mathbf{0} & \mathbf{B}_{2}
\end{array}\right] \cdots\left[\begin{array}{ll}
1 & \mathbf{c}_{n-1}^{T} \\
0 & \mathbf{B}_{n-1}
\end{array}\right]
$$

where $\mathbf{c}_{0}^{T}, \ldots, \mathbf{c}_{n-1}^{T}$ are row vectors, $\mathbf{0}$ denotes the zero vector, $\mathbf{I}$ the unit matrix and

$$
\mathbf{B}_{1} \mathbf{B}_{2} \ldots \mathbf{B}_{n-1}=\mathbf{S}^{n, q, r}=\mathbf{T}^{n, q, r}
$$


Also, the first column of $\mathbf{S}^{n+1, q, r}$ has 1 in the first row and zeros below. Thus it remains only to verify that the first rows of $T^{n+1, q, r}$ and $\mathbf{S}^{n+1, q, r}$ are equal. We have

$$
\left[S_{0,0}^{n+1, q, r}, \ldots, S_{0, n+1}^{n+1, q, r}\right]=\left[\mathbf{w}^{T}, 0\right]
$$

where, in the notation defined in the above lemma,

$$
\mathbf{w}^{T}=\mathbf{A}\left(1, q^{n}\right) \mathbf{A}\left(2, q^{n-1}\right) \ldots \mathbf{A}\left(n-1, q^{2}\right) \mathbf{A}(n, q) .
$$

In view of the lemma, we may permute the quantities $q^{n}, q^{n-1}, \ldots, q$ in (4.5), leaving $\mathbf{w}^{T}$ unchanged. In particular, we may write

$$
\mathbf{w}^{T}=\mathbf{A}\left(1, q^{n-1}\right) \mathbf{A}\left(2, q^{n-2}\right) \ldots \mathbf{A}(n-1, q) \mathbf{A}\left(n, q^{n}\right) .
$$

Now the product of the first $n-1$ matrices in (4.6) is simply the first row of $\mathbf{S}^{n, q, r}$ and thus

$$
\begin{aligned}
\mathbf{w}^{T} & =\left[S_{0,0}^{n, q, r}, \ldots, S_{0, n-1}^{n, q, r}\right]\left[\begin{array}{cccc}
1 & r^{n}-q^{n} & & \\
& \ddots & \ddots & \\
& & 1 & r-q^{n}
\end{array}\right] \\
& =\left[T_{0,0}^{n, q, r}, \ldots, T_{0, n-1}^{n, q, r}\right]\left[\begin{array}{cccc}
1 & r^{n}-q^{n} & & \\
& \ddots & \ddots & \\
& & 1 & r-q^{n}
\end{array}\right] .
\end{aligned}
$$

This gives

$$
S_{0,0}^{n+1, q, r}=T_{0,0}^{n, q, r}
$$

and

$$
S_{0 . j}^{n+1, q, r}=\left(r^{n+1-j}-q^{n}\right) T_{0, j-1}^{n, q, r}+T_{0, j}^{n, q, r}, \quad j=1, \ldots, n,
$$

noting that $T_{0, n}^{n, q, r}=0$. Then from (4.2)

$$
S_{0, j}^{n+1, q, r}=T_{0, j}^{n+1 . q, r}, \quad j=0, \ldots, n,
$$

and since $S_{0, n+1}^{n+1, q, r}=0=T_{0, n+1}^{n+1, q, r}$, the result is true for $n+1$ and the proof is complete.

The following is a consequence of Theorem 4.2 and Theorem 3.1.

Theorem 4.3. For $0<q \leq r^{n-1}$ the matrix $\mathbf{T}^{n, q, r}$ is totally positive.

We note that if $0<q \leq r^{n-1}$ and

$$
p=a_{0}^{q} P_{0}^{n, q}+\ldots+a_{n}^{q} P_{n}^{n, q}=a_{0}^{r} P_{0}^{n, r}+\ldots+a_{n}^{r} P_{n}^{n, r}
$$


then Theorem 3.2 shows that

$$
S^{-}\left(a_{0}^{r}, \ldots, a_{n}^{r}\right) \leq S^{-}\left(a_{0}^{q}, \ldots, a_{n}^{q}\right),
$$

see $\left[1\right.$, p. 166]. Since $\left(P_{0}^{n, 1}, \ldots, P_{n}^{n, 1}\right)$ is totally positive it follows from Theorem 3.3 that, for $0<q \leq r^{n-1} \leq 1$ and $p$ as in (4.7),

$$
S^{-}(p) \leq S^{-}\left(a_{0}^{r}, \ldots, a_{n}^{r}\right) \leq S^{-}\left(a_{0}^{q}, \ldots, a_{n}^{q}\right) .
$$

\section{Convexity}

From (4.8) we see that, for $0<q \leq 1, S^{-}\left(B_{n}^{q} f\right) \leq S^{-}(f)$. Since $B_{n}^{q}$ reproduces linear polynomials, this has the following consequence.

Theorem 5.1. For any function $f$ and any linear polynomial $p$,

$$
S^{-}\left(B_{n}^{q} f-p\right)=S^{-}\left(B_{n}^{q}(f-p)\right) \leq S^{-}(f-p),
$$

for $0<q \leq 1$.

This is illustrated by Figure 1. The function $f(x)$ is $\sin 2 \pi x$ and the generalized Bernstein polynomials are of degree $n=20$ with $q=0.8$ and $q=0.9$.

The next result follows from Theorem 5.1.

Theorem 5.2. If $f$ is increasing (decreasing) on $[0,1]$, then $B_{n}^{q} f$ is also increasing (decreasing) on $[0,1]$, for $0<q \leq 1$.

Proof. Let $f$ be increasing on $(0,1)$. Then, for any constant $c$,

$$
S^{-}\left(B_{n}^{q} f-c\right) \leq S^{-}(f-c) \leq 1
$$

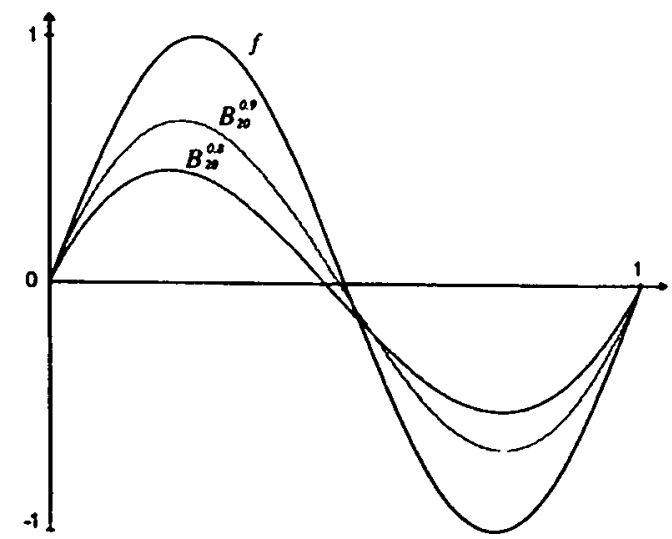

FIGURE 1: Sign changes of generalized Bernstein polynomials for $f(x)=\sin 2 \pi x$. The polynomials are $B_{20}^{0.8} f$ and $B_{20}^{0.9} f$. 
and thus $B_{n}^{q} f$ is monotonic. Since

$$
B_{n}^{q}(f ; 0)=f(0) \leq f(1)=B_{n}^{q}(f ; 1),
$$

$B_{n}^{q} f$ is monotonic increasing. (If $f$ is decreasing we may replace $f$ by $-f$.)

Next we recall the definition of a convex function.

Definition 5.1. A function $f$ is said to be convex on $[0,1]$ if, for any $t_{0}, t_{1}$ such that $0 \leq t_{0}<t_{1} \leq 1$ and any $\lambda, 0<\lambda<1, f\left(\lambda t_{0}+(1-\lambda) t_{1}\right) \leq \lambda f\left(t_{0}\right)+(1-\lambda) f\left(t_{1}\right)$.

Geometrically, this definition states that no chord of $f$ lies below the graph of $f$. We now state a result on convexity.

Theorem 5.3. If $f$ is convex on $[0,1]$, then $B_{n}^{q} f$ is also convex on $[0,1]$, for $0<q \leq 1$.

Proof. Let $p$ denote any linear polynomial. Then if $f$ is convex we have

$$
S^{-}\left(B_{n}^{q} f-p\right)=S^{-}\left(B_{n}^{q}(f-p)\right) \leq S^{-}(f-p) \leq 2 .
$$

Thus if $p(a)=B_{n}^{q}(f ; a)$ and $p(b)=B_{n}^{q}(f ; b)$ for $0<a<b<1$ then $B_{n}^{q} f-p$ cannot change sign in $(a, b)$. As we vary $a$ and $b$, a continuity argument shows that the sign of $B_{n}^{q} f-p$ on $(a, b)$ is the same for all $a$ and $b, 0<a<b<1$. From the convexity of $f$ we see that, when $a=0$ and $b=1,0 \leq p-f$, so that

$$
0 \leq B_{n}^{q}(p-f)=p-B_{n}^{q} f
$$

for $0<q \leq 1$ and thus $B_{n}^{q} f$ is convex.

We conclude this section by proving that, if $f$ is convex, the generalized Bernstein polynomials $B_{n}^{q} f$, for $n$ fixed, are monotonic in $q$.

Theorem 5.4. For $0<q \leq r \leq 1$ and for $f$ convex on $[0,1]$, then

$$
B_{n}^{r} f \leq B_{n}^{q} f
$$

Proof. Let us write $\zeta_{j}^{n, q}=\frac{[j]}{[n]}$ and $a_{j}^{n, q}=\left[\begin{array}{l}n \\ j\end{array}\right]$. Then, for any function $g$ on $[0,1]$,

$$
B_{n}^{q} g=\sum_{j=0}^{n} g\left(\zeta_{j}^{n, q}\right) a_{j}^{n, q} P_{j}^{n, q}=\sum_{j=0}^{n} \sum_{k=0}^{n} g\left(\zeta_{j}^{n, q}\right) a_{j}^{n, q} T_{j, k}^{n, q, r} P_{k}^{n, r}
$$

and thus

$$
B_{n}^{q} g=\sum_{k=0}^{n} P_{k}^{n, r} \sum_{j=0}^{n} T_{j, k}^{n, q, r} g\left(\zeta_{j}^{n, q}\right) a_{j}^{n, q}
$$


With $g=1$, this gives

$$
1=\sum_{j=0}^{n} a_{j}^{n, q} P_{j}^{n, q}=\sum_{k=0}^{n} P_{k}^{n, r} \sum_{j=0}^{n} T_{j, k}^{n, q, r} a_{j}^{n, q}
$$

and hence

$$
\sum_{j=0}^{n} T_{j, k}^{n, q, r} a_{j}^{n, q}=a_{k}^{n, r}, \quad k=0, \ldots, n .
$$

On putting $g(x)=x$ in (5.1), we obtain

$$
x=\sum_{j=0}^{n} \zeta_{j}^{n, q} a_{j}^{n, q} P_{j}^{n, q}=\sum_{k=0}^{n} P_{k}^{n, r} \sum_{j=0}^{n} T_{j, k}^{n, q, r} \zeta_{j}^{n, q} a_{j}^{n, q} .
$$

Since

$$
\sum_{j=0}^{n} \zeta_{j}^{n, r} a_{j}^{n, r} P_{j}^{n, r}=x
$$

we have

$$
\sum_{j=0}^{n} T_{j, k}^{n, q, r} \zeta_{j}^{n, q} a_{j}^{n, q}=\zeta_{k}^{n, r} a_{k}^{n, r}, \quad k=0, \ldots, n .
$$

Now if $f$ is convex, it follows from (5.2) and (5.3) that

$$
\begin{aligned}
f\left(\zeta_{k}^{n, r}\right) & =f\left(\sum_{j=0}^{n}\left(a_{k}^{n, r}\right)^{-1} T_{j, k}^{n, q, r} \zeta_{j}^{n, q} a_{j}^{n, q}\right) \\
& \leq \sum_{j=0}^{n}\left(a_{k}^{n, r}\right)^{-1} T_{j, k}^{n, q, r} a_{j}^{n, q} f\left(\zeta_{j}^{n, q}\right) .
\end{aligned}
$$

Then (5.1) gives

$$
\begin{aligned}
B_{n}^{q} f & =\sum_{j=0}^{n} f\left(\zeta_{j}^{n, q}\right) a_{j}^{n, q} P_{j}^{n, q} \\
& =\sum_{k=0}^{n} a_{k}^{n, r} P_{k}^{n, r} \sum_{j=0}^{n}\left(a_{k}^{n, r}\right)^{-1} T_{j, k}^{n, q, r} f\left(\zeta_{j}^{n, q}\right) a_{j}^{n, q} \\
& \geq \sum_{k=0}^{n} a_{k}^{n, r} P_{k}^{n, r} f\left(\zeta_{k}^{n, r}\right)=B_{n}^{r} f
\end{aligned}
$$




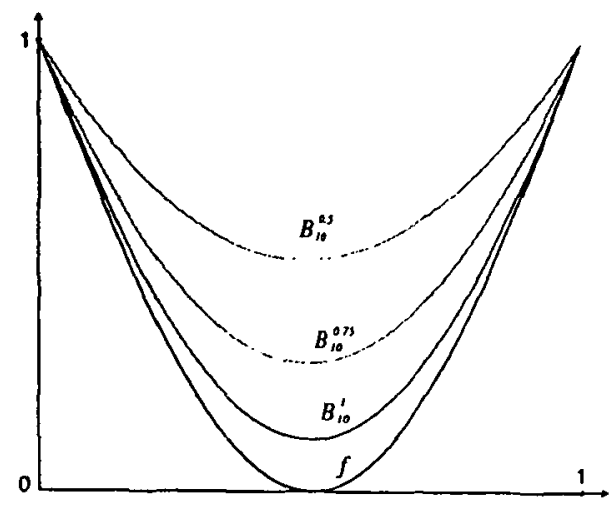

FIGURE 2: Monotonicity of generalized Bernstein polynomials in the parameter $q$, for $f(x)=1-\sin \pi x$. The polynomials are $B_{10}^{0.5} f, B_{10}^{0.75} f$ and $B_{10}^{1} f$.

Figure 2 illustrates the monotonicity in $q$ of the generalized Bernstein polynomials $B_{n}^{q}(f ; x)$ for the convex function $f(x)=1-\sin \pi x$.

\section{REFERENCES}

1. T. N. T. Goodman, Total positivity and the shape of curves, in Total Positivity and its Applications (M. Gasca, C. A. Micchelli (eds.), Kluwer, Dordrecht, 1996), 157-186.

2. S. L. Lee and G. M. Phillips, Polynomial interpolation at points of a geometric mesh on a triangle, Proc. Roy. Soc. Edinburgh 108A (1988), 75-87.

3. H. Oruç and G. M. Phillips, A generalization of the Bernstein polynomials, Proc. Edinburgh Math. Soc., to appear.

4. G. M. Phillips, Bernstein polynomials based on the q-integers, Ann. Numer. Math. 4 (1997), 511-518.

5. G. M. Phillips, A de Casteljau algorithm for generalized Bernstein polynomials, BIT 36 (1996), 232-236.

6. I. J. SChoenberg, On polynomial interpolation at the points of a geometric progression, Proc. Roy. Soc. Edinburgh 90A (1981), 195-207.

Tim N. T. GoOdman

Department OF MATHEMATICS AND

COMPUTER SCIENCE

UNIVERSITY OF DUNDEE

DUNDEE DD1 4 HN
Halil Oruç and George M. Phillips

Mathematical Institute

UNIVERSITY OF ST ANDREWS

NORTH HAUGH

ST ANDREWS

FIFE KY16 9SS 